\title{
Postdural Puncture Headache and Epidural Blood Patch in a Large Obstetric Anaesthesia Population
}

\author{
BM Serrano ${ }^{1}$, EC Cuenca ${ }^{1}$, AA Babarro ${ }^{1}$, EA Yanci $^{1}$, AS Dávila $^{1}$, EG Díaz $^{1}$, JD Sebastián ${ }^{2}$ and FG Rodríguez $^{1}$ \\ ${ }^{1}$ Hospital Universitario La Paz, Madrid, Spain \\ ${ }^{2}$ Department of Statistics, Hospital La Paz, Madrid, Spain
}

"Corresponding author: BM Serrano, Hospital Universitario La Paz, Madrid, Avenida de los Artesanos, 67, Tres Cantos, 28760, Madrid, Spain, Tel: 0034617363705; Email: blancabeatriz68@gmail.com

Received date: January 17, 2017; Accepted date: February 2, 2017; Published date: February 14, 2017

Copyright: ( 2017 Serrano BM, et al. This is an open-access article distributed under the terms of the Creative Commons Attribution License, which permits unrestricted use, distribution, and reproduction in any medium, provided the original author and source are credited.

\begin{abstract}
Background: Postdural puncture headache (PDPH) is the most common serious complication in obstetric anesthesia. We show the incidence of accidental dural puncture (ADP), PDPH, epidural blood patch (EBP) and the associated morbidity following an Obstetric Anesthesia Department's protocol.
\end{abstract}

Methods: An observational, prospective, analytical study was performed in 66.540 labor epidural analgesia. The objective is to describe the incidence of accidental dural puncture, postdural puncture headache and epidural blood patch in a large obstetric anesthesia practice population, as well as the associated morbidity of ADP and EBP.

Results: The incidence of accidental dural puncture obtained was $0.76 \%$, of postdural puncture headache $59 \%$, and the global incidence of epidural blood patch $0.2 \%$. Experience of the anesthetist performing the epidural (1st or 2nd year resident) and night time were associated with ADP. Low back pain rate was more frequent in those patients who received an epidural blood patch.

Conclusion: We found an incidence of ADP and PDPH of $0.76 \%$ and $59 \%$ respectively. Experience of the anesthetist performing the epidural (1st or 2 nd year resident) and nighttime were associated with ADP. EBP is a safe, easy and acceptable option as treatment for PDPH even with a higher risk of low back pain.

Keywords: Postdural puncture headache; Obstetric anesthesia; Accidental dural puncture; Epidural blood patch

\section{Introduction}

Accidental dural puncture (ADP) and subsequent postdural puncture headache $(\mathrm{PDPH})$ are known complications of neuraxial anesthesia. Despite the prevalence of this condition, relatively little is known about its pathophysiology, and most accepted treatments are not evidence-based. In obstetric patients, ADP occurs at a rate of $0.18 \%$ to $6 \%$ and approximately $50 \%$ to $70 \%$ of these develop PDPH $[1,2]$.

Strategies proposed to prevent PDPH after accidental dural puncture consider different measures including the insertion of epidural catheter intrathecally [3], early EBP [4] and epidural saline injection [5] that have shown limited efficacy. Little is known about the best prophylactic treatment for PDPH [6]. However, therapeutic epidural blood patch (EBP) is considered the gold standard for treatment [7].

Our aim was to describe the incidence and the risk factors of ADP, PDPH and EBP in a large obstetric anesthesia practice population, as well as the safety, feasibility and tolerability of the treatment with EBP. We present a written protocol for the management and monitoring of ADP populations.

\section{Materials and Methods}

We have performed a prospective, observational, analytical and descriptive study in our maternity of all the parturient that had undergone accidental Dural puncture during epidural block for labor analgesia. The study was carried out between June 2001 and October 2012 following the ethical committee approval (Hospital La Paz, Madrid; HULP code: PI-2107). These patients were managed using a standard departmental protocol for analgesia management after ADP and this is our institutional practice [Appendix 1].

\section{Inclusion and exclusion criteria}

Inclusion criteria for the study were:

I) Women with labor pain obtaining informed consent for epidural analgesia;

II) Parturients who delivered during this time period and had a known Dural puncture. The women under elective caesarean section with epidural anesthesia were excluded from the study.

\section{Protocol}

The epidural block was performed for women in labor. The epidural catheter was cited for labor analgesia and patients with scheduled caesarean section were excluded. The final delivery of pregnancy could either be vaginal, forceps or caesarean section. 
The main indications of caesarean section in these patients were cephalopelvic disproportion, failure of labor (the cervix has not dilated completely, labor has slowed or stopped, or the baby is not in an optimal delivery position) and fetal distress. The women under elective caesarean section are not included in this study.

Once the patient with labor pain was examined by the midwife, all procedures were performed with women in sitting position. ADP was defined as the objective loss of cerebrospinal fluid through the Tuohy needle when the anesthesiologists performing the technique.

Two sets were used for the technique's accomplishment; a Becton Dickinson ${ }^{\circledR}$ set (Perifase Plus KIT, Becton Dickinson and Company, MönlyckeHealt Care, Göteborg, Sweden) for epidural analgesia with a 18G Tuohy needle and a Braun Perifix ${ }^{\circ}$ set (Espocan+Docking system + Perifix soft tip, B, Braun, Melsungen, Germany) for combined epidural/intradural analgesia (18G Tuohy needle and 27G intradural needle). Epidural space was identified using the loss of resistance with air technique. Under a consultant supervision and methodology were performed by 1st year to 4th year anesthesia residents and by anesthesia staff. The 24 hours period into three groups: 8:00-15:00 (shift 1), 15:01-22:00 (shift 2) and 22:01-7:59 (shift 3).

Once ADP was recognized, the epidural catheter for labor analgesia was placed in a cephalic interspace in the first attempt and the catheter properly secured. By dispensing $3 \mathrm{~mL}$ of $0.25 \%$ bupivacaine with epinephrine the correct location of the epidural catheter was confirmed and the first analgesic dose was fractionally administered: 9 $\mathrm{ml}$ of $0.25 \%$ levo-bupivacaine and $20 \mu \mathrm{g}$ of fentanyl after which an epidural infusion pump (HospiraGemStar infusion pump blue) was connected $(0.125 \%$ levo-bupivacaine $+1.2 \mu \mathrm{g}$ fentanyl for each $\mathrm{mL}, 8$ $\mathrm{ml} / \mathrm{h}$ to $11 \mathrm{ml} / \mathrm{h}$ ) with the possibility of self-administered bolus (PCA: patient controlled analgesia: $8 \mathrm{ml}$ to $11 \mathrm{ml}, 15$ minute block range)

Regardless the mode of delivery (eutocic, instrumental o caesarean delivery), immediately after and also 12 hours to 20 hours later, an epidural injection of $20 \mathrm{ml}$ of $0.9 \%$ saline was administered, the epidural catheter was removed. No special management during the second stage of labor was considered in ADP women (not to have pushed, instrumental delivery to alleviate active pushing). During the first 24 hours after labor, postpartum analgesia was controlled with paracetamol $1 \mathrm{~g}$ IV every 6 hours and dexketoprofen trometamol 50 mg every 8 hours. Patients who underwent a caesarean section also received a bolus of $2 \mathrm{mg}$ of epidural morphine.

We used the International Headache Society definition of postdural puncture headache as a positional headache that worsened with sitting position and/or standing position, and that improved when adopting the decubitus supine position with at least one of five accompanying symptoms (nuchal rigidity, tinnitus, hypoacusia, photophobia, nausea, diplopia) [8]. During this period, PDPH was analysed. Data on the presence of associated symptoms were also recorded. If the conservative treatment failed, a therapeutic EBP for the management was offered to the patients. A written informed consent was obtained from the patients. After 48 hours of persistent PDPH with a VAS score $>7 / 10$, an epidural blood patch (EBP) was performed ( $15 \mathrm{ml}$ to $20 \mathrm{ml}$ or until the patient referred low back pain).

Women were kept supine for $1 \mathrm{~h}$. If the EBP was not efficient (patient continue suffering from headache during $48 \mathrm{~h}$ ), we would proceed to the performance of another EBP with the same method as the previous one. The 1st and the 2nd epidural puncture for EBP was performed by staff of the Anesthesia Department, preferably in the same intervertebral space where the dural puncture was produced. An additional image test (Magnetic Resonance) was required after 2nd unsuccessful EBP.

All the patients were phoned a week and three months later of the dural puncture and they were advised to contact the anesthesiology department if headache symptoms persisted in time. Morbidity originated as a consequence of the event was registered: low back pain, chronic headache and radiculopathy. We have also analysed the need of additional image tests as well as other symptoms like tinnitus or diplopia. Previous history of tensional headaches or migraines was registered. All the patients were instructed to contact the Department of Anesthesia if they developed or worse PDPH at any time thereafter.

\section{Statistical analysis}

For the description of quantitative continuous variables we used the mean with standard deviation. Categorical variables were described as absolute frequencies and relative frequencies were expressed in percentages. Comparison of quantitative variables between independent groups was conducted primarily using parametric tests (accomplishing the continuous criterium), using the Student $\mathrm{T}$ test when comparing two groups, or ANOVA analysis when three or more groups were involved. If, as a result of stratification, groups had 30 cases or less, statistical significance was obtained by non-parametric tests, Kruskal-Wallis or Mann-Whitney. Frequency analysis between qualitative variables was performed using the chi-square test or Fisher exact test when necessary (if $\mathrm{N}<20$, or if any value in the table of expected values was less than 5). We used the chi-square test with Yates correction.

\section{Results}

From the first of June 2001, until the 31st of October 2012, 66.540 epidural analgesia for labor were performed. We show the study flow chart in the (Figure 1).

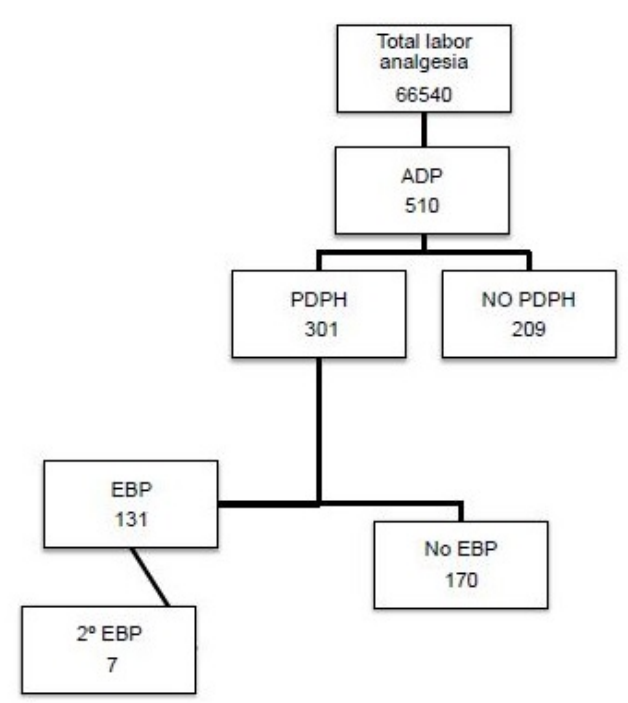

Figure 1: ADP: accidental dural puncture. PDPH: postdural puncture headache. EBP: epidural Blood patch. Total labor analgesia: all the women having epidural analgesia for labor pain. 
Citation: Serrano BM, Cuenca EC, Babarro AA, Yanci EA, Dávila AS,Díaz EG, et al. (2017) Postdural Puncture Headache and Epidural Blood Patch in a Large Obstetric Anaesthesia Population. J Pain Manage Med 3: 121.

Page 3 of 5

From the 66.540 epidurals performed, ADP occurred in 510, assuming a cumulative incidence of $0.76 \%$. Table 1 summarizes the demographic characteristics differences between total analgesic epidurals performed for labor versus those that result in accidental dural puncture.

ADP was more frequent in older patients with higher BMI $(\mathrm{p}=0.015)$ (Table 1).

\begin{tabular}{|c|c|c|c|c|c|c|}
\hline & $\begin{array}{l}\text { Total } \\
\text { Epidurals } \\
\text { mean(SD) }\end{array}$ & $\begin{array}{l}\text { Accidental } \\
\text { Dural } \\
\text { Punctures } \\
\text { mean (SD) }\end{array}$ & $\begin{array}{l}P \\
\text { value }\end{array}$ & $\begin{array}{l}\text { PDPH } \\
\text { mean } \\
\text { (SD) }\end{array}$ & $\begin{array}{l}\text { No } \\
\text { PDPH } \\
\text { mean } \\
\text { (SD) }\end{array}$ & $\begin{array}{l}P \\
\text { value }\end{array}$ \\
\hline Age & $30.6(5.5)$ & $\begin{array}{l}31.17 \\
(5.42)\end{array}$ & 0.022 & $\begin{array}{l}31.37 \\
(5.43)\end{array}$ & $\begin{array}{l}30.98 \\
(5.34)\end{array}$ & 0.421 \\
\hline Height & $\begin{array}{l}162.52 \\
(8.74)\end{array}$ & $\begin{array}{l}161.92 \\
(6.61)\end{array}$ & 0.125 & $\begin{array}{l}162.23 \\
(6.41)\end{array}$ & $\begin{array}{l}161.54 \\
(6.909)\end{array}$ & 0.252 \\
\hline Weight & $\begin{array}{l}74 \\
(11.33)\end{array}$ & $\begin{array}{l}74.04 \\
(12.36)\end{array}$ & 0.938 & $\begin{array}{l}73.65 \\
(11.93)\end{array}$ & $\begin{array}{l}74.57 \\
(12.97)\end{array}$ & 0.417 \\
\hline BMI & $\begin{array}{l}27.8 \\
(4.06)\end{array}$ & $\begin{array}{l}28.26 \\
(4.66)\end{array}$ & 0.015 & $\begin{array}{l}28.01 \\
(4.53)\end{array}$ & $\begin{array}{l}28.57 \\
(4.83)\end{array}$ & 0.192 \\
\hline $\begin{array}{l}\text { Cervical } \\
\text { Dilatation }\end{array}$ & $\begin{array}{l}3.98 \\
(1.58)\end{array}$ & $\begin{array}{l}4.07 \\
(2.02)\end{array}$ & 0.225 & $\begin{array}{l}4.03 \\
(1.97)\end{array}$ & $\begin{array}{l}4.13 \\
(2.05)\end{array}$ & 0.58 \\
\hline
\end{tabular}

BMI: Body Mass Index, SD: Standard deviation.

Table 1: Demographic data for total epidural analgesia and accidental dural punctures; $\mathrm{PDPH}$ population and NO PDPH population. Accidental dural punctures are included in total epidural analgesia.

We found no association between degree of cervical dilatation and the risk of ADP and PHPD, respectively. The mean value (SD) for cervical dilatation at epidural insertion was 3.81 (1.83). More ADP were performed by junior anesthesia residents $(p<0,001)$ at nighttime (shift 3) (Table 2).

\begin{tabular}{|l|l|l|l|}
\hline & NO ADP & ADP n (\%) & P value \\
\hline Shift 1 & 19797 & $160(0.8 \%)$ & \multirow{2}{*}{$\mathrm{P}=0.031$} \\
\cline { 1 - 3 } Shift 2 & 16453 & $143(0.87 \%)$ & \\
\cline { 1 - 3 } Shift 3 & 19670 & $207(1.05 \%)$ & \multirow{2}{*}{ P $<0.001$} \\
\hline Staff & 19304 & $108(0.56 \%)$ & \\
\hline 1st and 2nd YAR & 20432 & $216(1.05 \%)$ & \\
\hline 3nd and 4th YAR & 24003 & $181(0.75 \%)$ & \\
\hline
\end{tabular}

YAR: Year of anesthesia training. Shift $1(8: 00$ hours to $15: 00$ hours), shift 2 (15:01 hours to $22: 00$ hours) and shift 3 (22:01 hours to 7:59 hours). ADP: Accidental dural puncture.

Table 2: Shift and experience of the anesthetist performing the epidural related ADP.

A sum of $59 \%$ of patients who suffered ADP had PDPH. There were no statistical significant differences between the demographic characteristics of parturient that had suffered a PDPH and those who hadn't (Table 1).
Previous history of headache (migraine or tension headache) was not considered a risk factor for developing PDPH (59.9\% vs. 59.8\%, $\mathrm{p}=0.53$ ). The type of delivery wasn't either a risk factor as shown in (Table 3).

\begin{tabular}{|c|c|c|c|c|}
\hline \multicolumn{2}{|l|}{ Risk factors } & $\begin{array}{l}\text { NO PDPH \% } \\
(209 / 510)\end{array}$ & PDPH \% (301/510) & $P$ value \\
\hline \multicolumn{2}{|c|}{ Previous headache } & $40.20 \%$ & $\begin{array}{l}59.80 \% \\
(91 / 301)\end{array}$ & \multirow{3}{*}{0.537} \\
\hline \multicolumn{2}{|c|}{ Vaginal delivery } & $40.20 \%$ & $\begin{array}{l}59.80 \% \\
(217 / 301)\end{array}$ & \\
\hline \multicolumn{2}{|c|}{ Instrumental delivery } & $47.30 \%$ & $\begin{array}{l}52.70 \% \\
(30 / 301)\end{array}$ & \\
\hline \multirow[t]{2}{*}{ Caesareans } & $\begin{array}{l}\text { Fetal } \\
\text { distress }\end{array}$ & $34.30 \%$ & $\begin{array}{l}65.7 \% \\
(24 / 301)\end{array}$ & \multirow[t]{2}{*}{0.63} \\
\hline & Others & $40.90 \%$ & $59.1 \%(30 / 301)$ & \\
\hline
\end{tabular}

Both, fetal distress and other caesarean (all non-elective caesareans excluding fetal distress) have epidural catheter.

Table 3: Parturient suffering ADP and risk factors to developing $\mathrm{PDPH}$. Previous history of headache and PDPH, type of delivery and $\mathrm{PDPH}$

Out of the 510 ADP, 131 (25.7\%) received an EBP. From the total of patients who received an EBP, 5.3\% received a second because headache remained a problem. Overall EBP rate was $0.2 \%$.

Of the total parturient with severe PDPH (VAS $>7$ ), 37\% did not receive an EBP because they refused the technique and preferred to wait until the headache resolved. So, $72 \%$ of the women with headache lasting more than ten days did not receive an EBP (Figure 2).

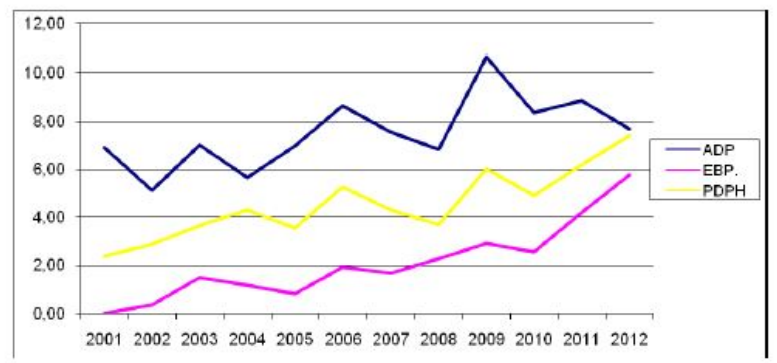

Figure 2: Incidence of ADP, PDPH and EBP/year, on the ordinate axis the data were in terms of rates per thousand. ADP: Accidental dural puncture. PDPH: Postdural puncture headache. EBP: epidural blood patch.

Long term back pain and chronic headache were the most frequent morbidity associated to the EBP population. There were no differences among side effects such as the incidence of additional imaging tests performed if parturient may have change in the characteristic of their headache, tinnitus or diplopia between patients who had received an EBP and those who had not (Table 4). 


\begin{tabular}{|l|l|l|l|}
\hline & $\begin{array}{l}\text { EBP } \% \\
(\mathbf{1 3 1 / 3 0 1})\end{array}$ & NO EBP \% & P value \\
\hline Chronic headache & $\begin{array}{l}6.10 \% \\
(9 / 131)\end{array}$ & $5.10 \%$ & 0.654 \\
\hline Low back pain & $\begin{array}{l}22.90 \% \\
(30 / 131)\end{array}$ & $3.50 \%$ & $<0.0001^{*}$ \\
\hline Radiculopaty & $\begin{array}{l}1.50 \% \\
(2 / 131)\end{array}$ & $0.50 \%$ & 0.276 \\
\hline Imaging & $\begin{array}{l}1.50 \% \\
(2 / 131)\end{array}$ & $0.30 \%$ & 0.16 \\
\hline Tinnitus & $\begin{array}{l}3.10 \% \\
(4 / 131)\end{array}$ & $2.90 \%$ & 0.57 \\
\hline Diplopia & $\begin{array}{l}1.50 \% \\
(2 / 131)\end{array}$ & $0.30 \%$ & 0.16 \\
\hline
\end{tabular}

EBP: Epidural blood patch.

Table 4: EBP and morbidity. Need of additional image tests and incidence of diplopia and tinnitus.

More patients with EBP were readmitted to the hospital (8.4\% vs. $0.5 \%, \mathrm{p}<0.001)$, mainly because of PDPH.

\section{Discussion}

We have studied a large population of patients who received epidural analgesia for labor and, specifically, those who suffered an ADP. The followed protocol perform a PDPH prophylaxis in all the adverted ADPs with two epidural saline patches, one of them at the end of expulsive period and the other 12 hours to 20 hours after birth. The incidence of ADP was $0.76 \%$ (66.540 epidural on labor).

Our results on the incidence of ADP are consistent with the literature $[1,2]$. Air was always used in the loss of resistance technique, even though differences of PDPH may occur if using saline. Results of other studies show that the risk of ADP was not related to the medium used for the loss of resistance technique [9].

We have found that body mass index (BMI) and age had a positive correlation with the rate of ADP. Faure et al. [10] showed that the incidence of ADP was approximately $4 \%$ in morbidly obese parturient compared to $0.25 \%$ to $0.5 \%$ in non-morbidly obese parturient $(\mathrm{p}<0.05)$.

It is important to point out that in 66.540 epidural procedures we have not found any association between degree of cervical dilatation and risk of ADP. However, we have found an increase in nocturnal risk of ADP and great evidence of relationship between experience of the anesthetist and occurrence of ADP $(\mathrm{p}<0.001)$, unlike Hollister [11].

Our findings have to be interpreted in the context that all the procedures were performed if the parturient referred labor pain in spite not having reported the mean VAS score at the time of epidural puncture.

The incidence of PDPH was 59\%; this is consistent with previous studies (50\% to $81 \%$ in pregnant women) [3]. No correlation between $\mathrm{BMI}$ and age with PDPH incidence was found, which is also consistent with other reports [12]. Hood et al. and Peralta et al. showed that the incidence of PDPH was significantly higher in non - morbidly obese parturient than in morbidly obese parturient but there is not no evidence for ADP and age [13,14].

The incidence of PDPH was not found to be different between women with and without previous migraine headache [15]. The type of delivery did not condition the appearance of PDPH, unlike other publications where the Valsalva maneuver worsened the headache [16]. Peralta et al. have suggested the association of pushing during labor and an increased likelihood of a PDPH after ADP [14].

Furthermore, patients who had a caesarean delivery were administered $2 \mathrm{mg}$ of epidural morphine, in order to control acute postoperative pain; however no significant difference in the PDPH rate was obtained with the use of epidural morphine [17].

The global incidence of EBP was $0.2 \%$. This result is lower than those describe by other studies $[10,13]$ which may be due to the use of prophylactic epidural saline injections during the postpartum period. The EBP rate in patients with ADP was $25.7 \%$ and when necessary a second EBP 5.3\%; this correlates with the work by Baysinger et al. who found similar results [18]. In addition, $37 \%$ of the patients with severe PDPH declined the EBP despite a VAS score $>7 / 10$.

The fact that few institutions have a written protocol for managing ADP is indicative of the wide variation in the management of ADP and PDPH and also of the lack of evidence available for PDPH prevention. In our protocol the EBP has always been used as PDPH treatment and never as prophylaxis [19]. It has always been always performed 48 hours after the event, due to the fact that higher failure rates have been reported with early rather than delayed performance [20].

Nowadays the perfect prevention of PDPH after an ADP does not exist. Many alternatives are offered without great results [21]. One of the methods used in the PDPH treatment is the intrathecal insertion of an epidural catheter, but significant controversy exits in the literature regarding the safety and efficacy of this modality. Early EBP has been associated to neurological complications [22]. Some authors have demonstrated that epidural saline injections have a lower success rate than the EBP, but on the other hand, other studies suggest that the epidural saline patch is an alternative strategy to EBP for PDPH; there is no clear consensus about which prophylactic measure is the most effective.

The most aggressive measure within the protocol was the performance of EBP; that is the reason we studied the long-term repercussions of this therapeutic measure. In relation to morbidity, after phonic call follow at ten days and three months after the procedure, low back pain was the most common finding. With great statistical significance, it has been more frequent in the patients who received an EBP, unlike to what has been published by other authors, who claim that the incidence of low back pain was not increased in women who have received EBP [23]. Our data correlates with the work by Kakinohana et al. [22] who reported that low back pain was more frequent in the patients who received EBP. Low back pain is something that can occur in postpartum women who did not receive epidural analgesia; this is the reason why these results should be managed with caution. Hospital readmission rate was higher in patients who received an EBP, not being so regarding the incidence of diplopia, tinnitus or need of complementary imaging tests due to an erratic course of PDPH [24].

With these data and the implementation of a protocol [25], we would like to emphasize that during the follow up done to these 
patients, with two epidural saline patches and 131 EBP made, we had no serious complications, but we obtained additional low back pain (23\%) in the EBP group.

This study has several limitations; firstly, our study is observational. Second, physicians performing the technique had different degrees of experience, ranging from anesthesia residents to obstetric anesthesia attending, which could be considered a bias; nevertheless, we tried to reproduce the normal conditions in clinical practice.

We believe that there are different clinical circumstances in which ADP occurs, but his study is one of the largest studies performed in women with labor pain, ADP and EBP following a written protocol to optimize and monitor the associated morbidity.

Clinical implications are about the incidence of ADP (despite working in a teaching hospital, our results indicate low incidence of ADP), about the EBP morbidity (with high size of EBP patients) and finally, about the management of ADP according a protocol [26].

\section{Conclusion}

We found an incidence of ADP and PDPH of 0.76\% and 59\% respectively. Experience of the anesthetist performing the epidural (1st or 2nd year resident) and nighttime were associated with ADP. Implementation of standardized protocols is of maximum importance to monitor and manage ADP and PDPH. EBP is a safe, easy and acceptable option as treatment for PDPH even with a higher risk of low back pain.

\section{Acknowledgement}

The first author would like to thank all the obstetric anesthesia staff and the residents.

\section{References}

1. Banks S, Paech M, Gurrin L (2001) An audit of epidural blood patch after accidental dural puncture with a Tuohy needle in obstetric patients. Int J Obstet Anesth 10: 172-176.

2. Heesen M, Klöhr S, Rossaint R, Van De, Velde M, Straube S (2013) Can the incidence of accidental dural puncture in laboring women be reduced? A systematic review and meta-analysis. Minerva Anestesiol 79: 1187-1197.

3. Kaddoum R, Motlani F, Kaddoum RN, Srirajakalidindi A, Gupta D, et al. (2014) Accidental dural puncture, postdural puncture headache, intrathecal catheters, and epidural blood patch: revisiting the old nemesis. J Anesth 28: 628-630.

4. Sudlow C, Warlow C (2010) Epidural blood patching for preventing and treating post-dural puncture headache. Cochrane Database Syst Rev 1: CD001791.

5. Kiki I, Gundogdu M, Alici HA, Yildirim R, Bilici M (2009) A simple, safe and effective approach to prevent postdural puncture headache: epidural saline injection. Eurasian J Med 41: 175-179.

6. Bradbury CL, Singh SI, Badder SR, Wakely LJ, Jones PM (2013) Prevention of post-dural puncture headache in parturients: a systematic review and meta-analysis. Acta Anaesthesiol Scand 57: 417.

7. Buettner A, Popham P, Morgan D (2005) Incidence of epidural blood patch following obstetric regional analgesia in private Australian anaesthetic practice. Int J Obstet Anesth 14: 5-8.
8. https://www.ichd-3.org/7-headache-attributed-to-non-vascularintracranial-disorder/7-2-headache-attributed-to-low-cerebrospinalfluid-pressure/7-2-1-post-dural-puncture-headache/

9. Schier R, Guerra D, Aguilar J, Pratt GF, Hernández M, et al. (2009) Epidural space identification: a meta-analysis of complications after air versus liquid as the medium for loss of resistance. Anesth Analg 109: 2012-2021.

10. Faure E, Moreno R, Thisted R (1994) Incidence of postdural puncture headache in morbidly obese parturients. Reg Anesth 19: 361-363.

11. Hollister N, Todd C, Ball S, Thorp-Jones D, Coghill J (2012) Minimising the risk of accidental dural puncture with epidural analgesia for labour: a retrospective review of risk factors. Int J Obstet Anesth 21: 236-241.

12. Miu M, Paech MJ, Nathan E (2014) The relationship between body mass index and post-dural puncture headache in obstetric patients. Int J Obstet Anesth 23: 371-375.

13. Hood DD, Dewan DM (1993) Anesthetic and obstetric outcome in morbidly obese parturients. Anesthesiology 79: 1210-1218.

14. Peralta F, Higgins N, Lange E, Wong CA, McCarthy RJ (2015) The Relationship of Body Mass Index with the Incidence of Postdural Puncture Headache in Parturients. Anesth Analg 121: 451-456.

15. Van Oosterhout WP, van der Plas AA, van Zwet EW, Zielman R, Ferrari $\mathrm{MD}$, et al. (2013) Postdural puncture headache in migraineurs and nonheadache subjects: a prospective study. Neurology 80: 941-948.

16. Angle P, Thompson D, Halpern S, Wilson DB (1999) Second stage pushing correlates with headache after unintentional dural puncture in parturients. Can J Anaesth 46: 861-866.

17. Cesur M, Alici HA, Erdem AF, Silbir F, Celik M (2009) Decreased incidence of headache after unintentional dural puncture in patients with cesarean delivery administered with postoperative epidural analgesia. J Anesth 23: 31-35.

18. Baysinger CL, Pope JE, Lockhart EM, Mercaldo ND (2011) The management of accidental dural puncture and postdural puncture headache: a North American survey. J Clin Anesth 23: 349-360.

19. Stein M (2014) Prophylactic versus therapeutic blood patch for obstetric patients with accidental dural puncture-a randomized controlled trial. Anaesthesia 69: 320-326.

20. Kokki M, Sjövall S, Keinänen M, Kokki H (2013) The influence of timing on the effectiveness of epidural blood patches in parturients. Int J Obstet Anesth 22: 303-309.

21. Verstraete S, Walters MA, Devroe S, Roofthooft E, Van de, Velde (2014) Lower incidence of post-dural puncture headache with spinal catheterization after accidental dural puncture in obstetrics patients. Acta Anaesthesiol Scand 58: 1233-1239.

22. Kakinohana M, Odo Y, Matsuda S, Taira Y, Sugahara K (2001) Epidural injection with saline for treatment of postspinal headache: comparison with epidural blood patch. J Anesth 15: 185-187.

23. Vercauteren MP, Hoffmann VH, Mertens E, Sermeus L, Adriaensen HA (1999) Seven-year review of requests for epidural blood patches for headache after dural puncture: referral patterns and the effectiveness of blood patches. Eur J Anaesthesiol 16: 298-303

24. Bleeker CP, Hendriks IM, Booij LH (2004) Postpartum post-dural puncture headache: is your differential diagnosis complete? Br J Anaesth 93: 461-464.

25. Ayad S, Demian Y, Narouze SN, Tetzlaff JE (2003) Subarachnoid catheter placement after wet tap for analgesia in labor: influence on the risk of headache in obstetric patients. Reg Anesth Pain Med 28: 512-515.

26. Gaiser RR (2013) Postdural puncture headache: a headache for the patient and a headache for the anesthesiologist. Curr Opin Anaesthesiol. 26: 296-303. 